\title{
A Noncontact Technique to Measure HRV
}

\author{
P. H. de B. Souza \\ PUC Goiás \\ Av. Universitaria 1.440 \\ Goiânia/GO, Brazil
}

\author{
J. O. Ferreira \\ PUC Goiás \\ Av. Universitaria 1.440 \\ Goiânia/GO, Brazil
}

\author{
A. F. Rocha \\ University of Brasília \\ Campus D. Ribeiro \\ Brasília/DF, Brazil
}

\author{
T. M. Barbosa \\ PUC Goiás \\ Av. Universitaria 1.440 \\ Goiânia/GO, Brazil
}

\begin{abstract}
The heart rate and its variability, known as Heart Rate Variability (HRV), are indispensable measurements for cardiorespiratory monitoring, recognition and quantification of emotions, detection of abnormalities, and heart disease control. In general, the acquisition systems for heart rate and HRV require a contact area for sensor's installation and positioning, creating restrictions and/or obstructions on user's movements. This paper proposes a noninvasive and noncontact technique for heart rate and HRV acquisition using a single camera. This method consists in the automatic detection of the user's face and utilization of an Independent Component Analysis (ICA) algorithm to separate the necessary signals to determine the heart rate and the HRV. The experiments have shown more than $80 \%$ of similarity between the results of the proposed heuristic in comparison to the results of the photoplethysmography sensor (PPG).
\end{abstract}

\section{General Terms}

Biofeedback, Image Processing.

\section{Keywords}

Noncontact technique, heart rate, HRV, camera.

\section{INTRODUCTION}

The HRV is an important measurement in the field of psychophysiology. It is commonly used to detect the activity of two branches of the Autonomic Nervous System (ANS), the Sympathetic Nervous System (SNS) and Parasympathetic Nervous System (PNS), which have influence in the heartbeat intervals [1]. Considering the importance of HRV, Picard et $a l$. proposed a robust method to measure the heart rate using a digital camera and the ambient light [2]. This technique consists in detecting the blood volume pressure (BVP) in the human face through the variation caused by the heartbeats. The HRV signals have a huge potential because they can provide important data about the cognitive and emotional states of the user [3]. In addition, the analysis of the HRV can determinate changes in the user's heartbeat, which can characterize heart diseases, such as hypertension [4][5].

This paper proposes and describes a heuristic to quantify automatically the HRV based on the heartbeat intervals. This heuristic was evaluated using the HRV results of a PPG sensor embedded in a device designed for entertainment, known as Affective Gamepad [6].

\section{MATERIALS AND METHODS}

The experiments were conducted in a laboratory with controlled light intensity $(200 \mathrm{~cd})$ with a small incidence of sunlight entering through the windows. Twenty Computer Engineering students of the Pontifical Catholic University of Goias, of both genders, different ages $(18-40$ years old), and color skin were invited to test the prototype. During the tests, the students were asked to keep still and face the camera while one minute of video was being recorded. At same time, the Affective Gamepad captured the HRV and heart rate signals using its PPG sensor. These signals are used to validate the HRV and heart rate obtained by the camera. The samples were obtained as shown in Figure 1. The computer responsible for running the application was equipped with an Intel Core i7 4710HQ 2.5GHz, with 16GB of RAM.

A MATLAB software was written to analyze the videos. The Figure 2 describes the entire process to recover the heart rate and the HRV. First, it was utilized the Computer Vision System Toolbox to identify and track the user's face. After identifying the user's face in a frame of the video, only the forehead and cheek regions were selected to compose the region of interest (ROI). It avoids the movements of the eyes and mouth because they would increment noise in the results. Each ROI was separated in RGB channels and the mean of all pixels of each channel was calculated in order to create the raw signals. The raw signals are the base for the heart rate and HRV detection, and they consist in $\mathrm{XR}(\mathrm{t}), \mathrm{XG}(\mathrm{t})$ and $\mathrm{XB}(\mathrm{t})$, representing the red, green, and blue channels, respectively.

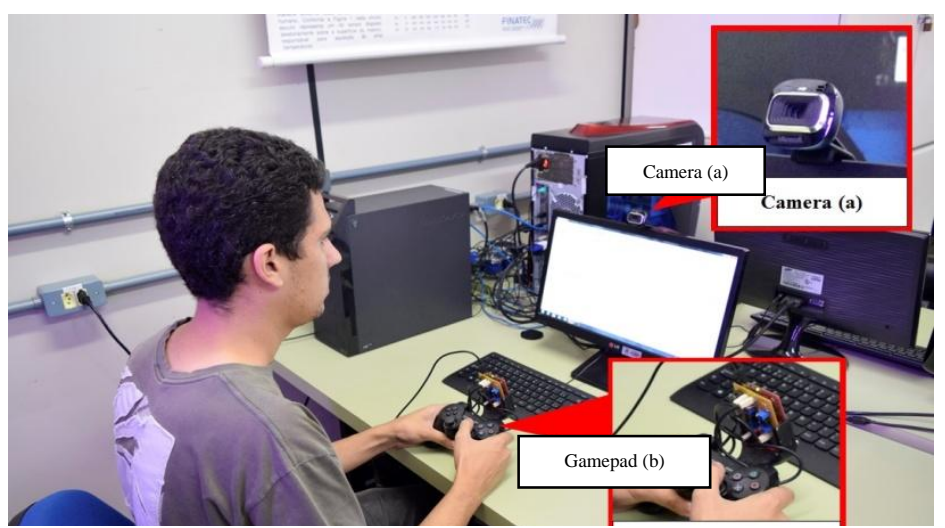

Fig. 1. (a) Microsoft LifeCam from a distance of $50 \mathrm{~cm}$ of the user, recording a video in high definition $(1280 \times 720 \mathrm{p}$, 24-bit, 30 fps) of the user's face. (b) Gamepad capturing the heart rate and the HRV using the PPG sensor 
(b)

(a)

(c)

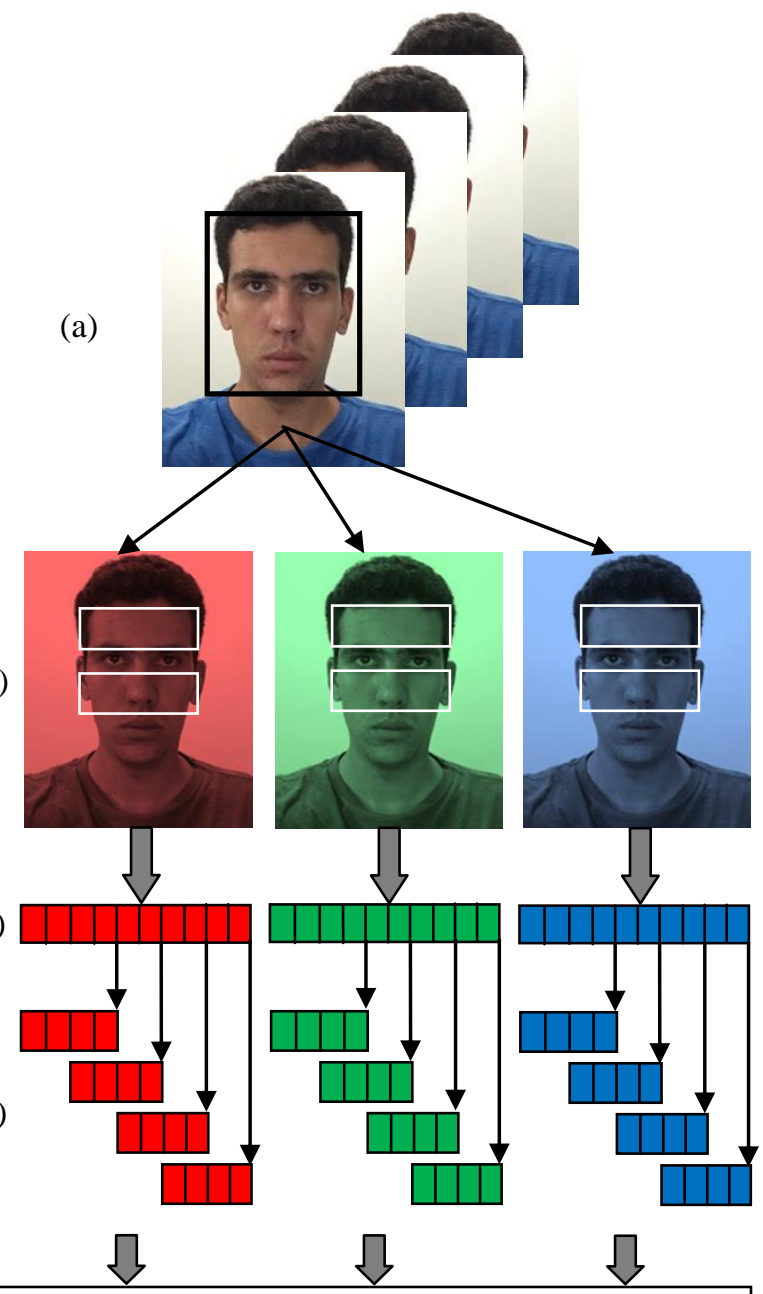

(e) Independent Component Analysis

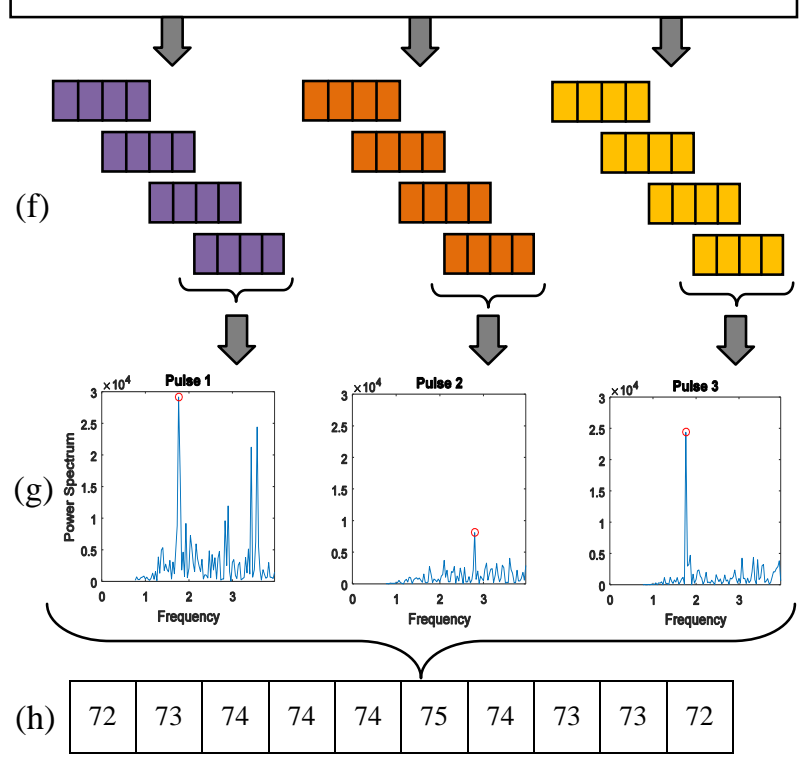

Fig 2. Recovery of heart rate and HRV components. This diagram shows how the experiment was conducted. (a) Face detection in each frame. (b) Only the forehead and cheek are selected to be the ROI in each color channel. (c) Mean of
ROI, creating the RGB raw signals. (d) The raw signals are separated into blocks, increasing the number of HRV samples. (e) The components are separated using ICA. (f) Three resulting components of ICA. (g) Power spectrum analysis of the three components. The highest peak is chosen to be an element of the heartbeat frequencies vector. (h) Heartbeat Frequencies in Hertz (Hz).

In the experiments, 60 seconds of video were recorded, at 30 frames per second (fps), resulting in raw signals with 1800 samples. After this process, the video was separated into blocks that were posteriorly processed, creating the heart rate and HRV signals. The block overlap and length could be adjusted in order to increase or decrease the dependency between each block. For example, if the overlap is 9 and the length is 10 , the first block represents the interval between 1 and 10 seconds, the second block represents the interval between 2 and 11 seconds, and so on. Then, each block was detrended using the smoothness priors approach [7], with a smooth parameter $\lambda=10$, removing the low frequency components that are not necessary to recover the heart rate and the HRV. The resulting blocks were normalized subtracting the mean and dividing by the standard deviation.

After detrending and normalizing the signals of each block, the Independent Component Analysis (ICA) technique was applied over the raw signals [8], creating the independent components that were posteriorly analyzed using a power spectrum approach. Only the frequencies between $0.75 \mathrm{~Hz}$ and $4 \mathrm{~Hz}$ were considered, because they are the acceptable range of the human heartbeat [2]. The most powerful frequency between the three resulting signals is the heart rate value of the block (in Hz). To obtain the heart rate in beats per minute (BPM), the heart rate values are multiplied by 60 .

\section{RESULTS}

Using the technique described in Materials and Methods, the heart rate and the HRV were recovered from the videos using the ICA algorithm. The results of the experiment with a 22 years old white man are shown in Table 1 .

Table 1. Overall Results

\begin{tabular}{|c|c|c|c|}
\hline Blocks & $\begin{array}{c}\text { Blocks Intervals } \\
\text { (seconds) }\end{array}$ & $\begin{array}{c}\text { Heart Beat } \\
\text { Frequency (Hz) }\end{array}$ & $\begin{array}{c}\text { Heart Rate } \\
\text { (BPM) }\end{array}$ \\
\hline 1 & $1-15$ & 1 & 60 \\
\hline 2 & $7-21$ & 1 & 60 \\
\hline 3 & $13-27$ & 1 & 60 \\
\hline 4 & $19-33$ & 1 & 60 \\
\hline 5 & $25-39$ & 1.05 & 63 \\
\hline 6 & $31-45$ & 1.08 & 65 \\
\hline 7 & $37-51$ & 1.13 & 68 \\
\hline 8 & $43-57$ & 1.26 & 76 \\
\hline 9 & $49-60$ & 1.2 & 72 \\
\hline
\end{tabular}

In the Table 1, the second column represents the intervals of the blocks created during the software processing. In this experiment, the blocks had an overlap of 9 seconds and length equals to 15 seconds. The length and the overlap are adjusted according to the heart rate results obtained by the Affective 

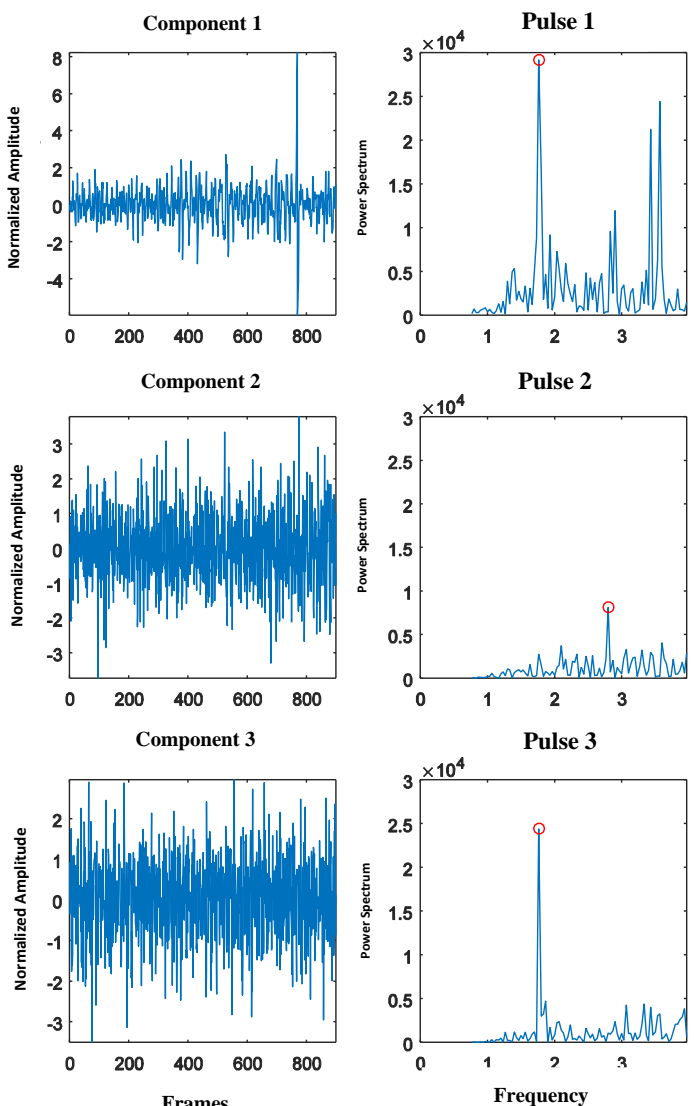

Fig. 3. Pulse 1 graphic represents the power spectrum of the first component of the raw signals. The higher peak in Pulse 1 (red circle) is also the higher peak between the other two power spectrum (Pulse 2 and Pulse 3 graphics respectively). Pulse 1 determined the heart rate of the last block captured by the camera.

Gamepad. When the values of the heart rate obtained by the camera approximate to the values obtained by the Affective Gamepad, it is established the ideal values for the software calibration. These values can be different depending on the user because of the different skin colors and user's face format. The third and fourth columns show the values of heart rate along one minute of video. The Figure 3 shows the ICA components of the last block and the higher peak frequency in each pulse. The higher peak between those three was used to obtain the HRV.

\section{DISCUSSION}

At this stage of development, the described technique is running offline. This means that the video is first recorded and the heart rate and HRV detections are done posteriorly. The difference between the project described in this paper and the method developed by Picard et al. [2] is how the software handles the user's video. Working with blocks gives the possibility of adapting it to work with real-time feedback (online), providing the heart rate and the HRV while the video is being recorded.

In order to compare the results of the camera with the results of the Affective Gamepad, the user's videos and samples of the PPG were recorded at same time. The Figure 4 shows the results of this comparison.
Test 1
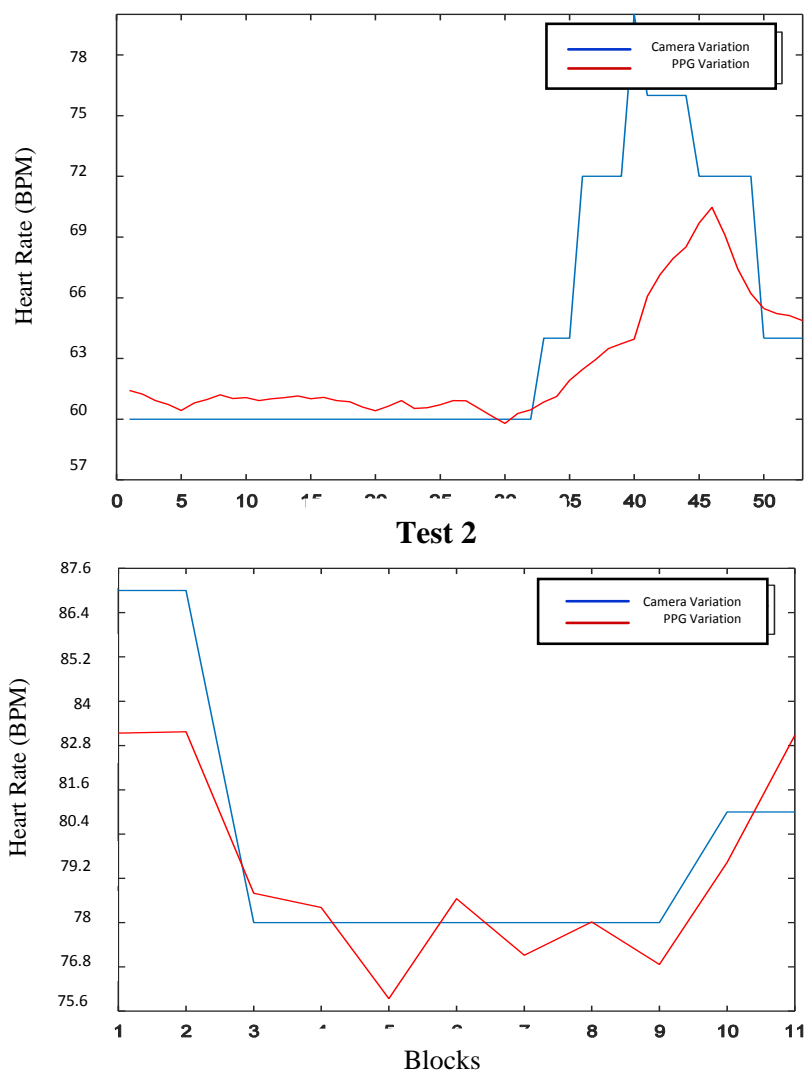

Fig. 4. Comparison between the HRV obtained by the camera and the HRV obtained by the PPG. The Test 1 shows the HRV results to an experiment with 53 blocks of video, while the Test 2 shows the results to 11 blocks. The calibration established the values of length and overlap of each block

To measure the similarity of the signals, it was utilized the Pearson Correlation Coefficients technique [9]. This technique consists in measuring the direction and the degree of linear relationship between the HRV signals of the camera and the Affective Gamepad. In Test 1, the HRV taken by the camera obtained $86 \%$ of similarity with the HRV obtained by the PPG, while in Test 2, the signals obtained $84 \%$ of similarity. These values prove that there is a high degree of linear relationship between those signals.

In both tests, the users did not suffer any external stimulus. They only reported what they felt during the test. The user (Test 1) reported that in the beginning of the test he was calm, and he started to be anxious after 45 seconds of recording. The user's anxiety induced the increase in the heart rate, as shown in the Test 1 graphic. The other user (Test 2) reported that he was calm during the test. However, the camera and the PPG detected the same variations in the user's heart rate. On both tests, the heart rate obtained by the PPG varied more than the heart rate obtained by the camera. This happened because of the movement of the user's hands during the test.

\section{CONCLUSION}

There are other techniques, such as video magnification [10], that are commonly used to measure the heart rate and its variation using cameras. These techniques are part of applications developed for mobile devices, due their high 
efficiency in terms of processing and memory use. However, the ICA technique resulted in heart rate values closer to the values obtained by the PPG [2][10]. During the tests, the ICA technique required less than 1 second to process 1 minute of video. This allows the adoption of this technique in mobile devices, which have limited processors in comparison to desktop computers. The application responsible for running this technique would evaluate the user's effort and attention in background and in parallel with other applications.

Future work will be focused on adapting this algorithm to be part of a framework that will be used in mobile applications. Applications using this framework will be able to get real-time feedback of heart rate and HVR signals.

\section{ACKNOWLEDGMENT}

This study was supported by FAPEG (in Portuguese, Fundação de Amparo à Pesquisa do Estado de Goiás) at Pontifical Catholic University of Goiás.

\section{REFERENCES}

[1] D. J. McDuff, S. Gontarek, and R. W. Picard, "Remote measurement of cognitive stress via heart rate variability." Engineering in Medicine and Biology Society (EMBC), 2014 36th Annual International Conference of the IEEE. IEEE, 2014.

[2] M.-Z. Poh, D. J. McDuff, and R. W. Picard, "Advancements in noncontact, multiparameter physiological measurements using a webcam," Biomedical Engineering, IEEE Transactions on, vol. 58, no. 1, pp. 7-11, 2011.

[3] D. J. McDuff, S. Gontarek, J. Hernandez, and R. W. Picard. "COGCAM: Contact-free Measurement of
Cognitive Stress During Computer Tasks with a Digital Camera". Media Lab, Massachusetts Institute of Technology, 2016.

[4] H. Halik e A. Johncamm. "Heart Rate Variability". Futura Publishing Company, 1995.

[5] M. Malik. "Heart Rate Variability, Standards of Measurement, Physiological Interpretation, and Clinical Use." Writing Committee of the Task Force, Department of Cardiological Sciences, St. George's Hospital Medical School, Cranmer Terrace, London, U.K, 1996.

[6] P. H. F. Bacchini, E. C. Lopes, M. A. Barbosa, J. O. Ferreira, O. C. S. Neto, A. F. Rocha, and T. A. Barbosa. "Developing an Affective Point-of-Care Technology". Symposium Series on Computational Intelligence (SSCI). IEEE, 2014.

[7] M. P. Tarvainen, P.O. Ranta-Aho, and P.A. Karjalainen, "An advanced detrending method with application to HRV analysis," IEEE Trans. Biomed. Eng., vol. 49, no. 2, pp. 172-175, Feb. 2002.

[8] P. Comon, "Independent component analysis, a new concept?” Signal Process, vol.36, pp.287-314, 1994.

[9] D. Figueiredo e J. Silva. "Desvendando os Mistérios do Coeficiente de Correlação de Pearson." V Seminário de Ciência Política e Relações Internacionais da Universidade Federal de Pernambuco. Revista Política Hoje, Vol. 18, n. 1, 2009.

[10] M. Estrada, A. Stowers, "Amplification of Heart Rate in Multi-Subject Videos". [Online]. Available in: https://web.stanford.edu/class/ee368/Project_Spring_141 5/Reports/Stowers_Estrada.pdf 